SHORT REPORT

\title{
Randomised control trial of tongue acupuncture versus sham acupuncture in improving functional outcome in cerebral palsy
}

\author{
J G Sun, C H Ko, V Wong, X R Sun
}

J Neurol Neurosurg Psychiatry 2004;75:1054-1057. doi: 10.1136/innp.2003.021485

Background: Traditional Chinese medicine (TCM) has been used historically in the treatment of cerebral palsy (CP). We investigated the efficacy of acupuncture in improving the motor function of children with CP.

Methods: A randomised control trial was conducted to assess the effect of tongue acupuncture (TAC) in $33 \mathrm{CP}$ children. The subjects were randomised to treatment $(n=22)$ with TAC or control $(n=11)$. Clinical outcome was evaluated using the gross motor function measure (GMFM) and the pediatric evaluation of disability inventory (PEDI).

Results: The increase in mean GMFM score was significantly greater in the treatment than in the control group ( $p=0.042$ ). Conclusion: An improvement in motor function of $\mathrm{CP}$ subjects is seen following a short course of acupuncture.

$\mathrm{R}$ habilitation management programs for subjects with cerebral palsy (CP) aim to achieve functional improvement, especially using new treatments such as botulinum toxin, selective dorsal rhizotomy, or intrathecal baclofen infusion. ${ }^{1-3}$ As CP is a chronic neurological condition, parents tend to seek alternative treatments such as acupuncture. Anecdotal reports of body and scalp acupuncture for CP have demonstrated its efficacy. ${ }^{4-9}$ However, evidence-based investigation assessing the efficacy of acupuncture in $\mathrm{CP}$ is lacking.

According to the philosophy of traditional Chinese medicine (TCM), health is achieved by maintaining an uninterrupted flow of "Qi" along 14 meridians. Disease is caused by stagnation of the flow of this "Qi" or energy. Acupuncture can help to restore the smooth flow of Qi, thus restoring the internal balance. ${ }^{10}$

In TCM, the tongue is regarded as the window for diagnosis as it has rich nerve and blood supplies. Tongue acupuncture (TAC) was invented by the first author based on experience in treating chronic neurological diseases. We postulate that there are acupoints in the tongue linked to the 14 meridians. The physiological effect might be based on resignalling or potentiation of neural receptors through repeated stimulation. As the tongue is close to the brainstem and cerebellum, we postulate that stimulating tongue acupoints might augment the neural pathways connected to the motor/somatosensory cortex, resulting in improvement in motor function. We have demonstrated improvement in drooling in CP with a short course of TAC. ${ }^{11}$

The objective of this study was to assess whether a short course of TAC could improve motor function of CP children.

\section{METHOD}

A randomised control trial was conducted from April 2000 to January 2001. CP children attending the Children's
Habilitation Institute of the Duchess of Kent Children's Hospital of the University of Hong Kong were invited to participate in this research after full explanation to their parents. This study was approved by the Ethics Committee of the Faculty of Medicine of the University of Hong Kong. Written informed consent was obtained from parents.

A total of $33 \mathrm{CP}$ children ( 16 boys, 17 girls) were recruited. The treatment $(\mathrm{T})$ and control $(\mathrm{C})$ groups were matched for age, sex, and GMFM and PEDI scores.

The treatment $(\mathrm{T})$ group $(\mathrm{n}=22$ )

The mean (SD) age of the treatment group (eight boys, 14 girls) was 8.62 (3.50) (range: 3.52-16.80) years. Impairment consisted of diplegia (9), hemiplegia (6), bilateral hemiplegia (3), triplegia (1), dyskinesia (2), and ataxia (1). Three subjects had moderate grade mental retardation (MR), six had mild MR, and 13 had normal intellectual function. Thirteen were ambulatory, and nine were partially ambulatory.

The control $(C)$ group $(n=11)$

The mean (SD) age of the control group (eight boys, three girls) was 10.68 (3.13) (range: 4.47-14.09) years. Impairment included spastic diplegia (8), hemiplegia (1), and bilateral hemiplegia (2). Two subjects had moderate grade MR, two had mild MR, and six had normal intellectual function. Four were ambulatory and seven were partially ambulatory.

\section{Randomisation process}

We use random permuted blocks to maintain a ratio of $2: 1$. The principle investigator (VW), clinical evaluator (CHK), parents, and their children were blind to the randomisation list and only the acupuncturist (JGS) knew the treatment allocation. The code was broken upon completion of the trial and data entry. All children continued their physiotherapy program in the special training centres or special schools.

\section{Method of tongue acupuncture}

Patients allocated to the treatment group received 40 sessions of TAC on a daily basis conducted over 8 weeks. The acupuncture lasted for less than $15 \mathrm{~s}$ and no sedation was required. The child was allowed to sit on the mother's lap with the head tilted $45^{\circ}$ upwards and was encouraged to open his/her mouth. Sterile gauze was used to pick up and station the tongue with the acupuncturist's left hand and quick and accurate insertion into acupoints was performed with the right. We used sterile disposable acupuncture needles, $4 \mathrm{~cm}$ in length and gauge $30(0.3 \mathrm{~mm})$ (HWA TO, made in China).

Based on our clinical experience of more than $100 \mathrm{CP}$ children in China, three acupoints were used: (a) two

Abbreviations: CP, cerebral palsy; GMFM, Gross Motor Function Measure; MR, mental retardation; PEDI, Pediatric Evaluation of Disability Inventory; TAC, tongue acupuncture; TCM, traditional Chinese medicine 
acupoints on the lateral surface of the tongue, known as Xian Zuo (the midline between the tip and root of the left lateral surface of the tongue, TAC no 1) and Xian You (the midline between the tip and root of the right lateral surface of the tongue, TAC no 2) and (b) one acupoint at the tongue base, known as Di Men (the midline of tongue base, TAC no 3 ). Both TAC no 1 and no 2 were punctured obliquely at $45^{\circ}$ to a depth of $0.3-0.5 \mathrm{~cm}$ and TAC no 3 was punctured perpendicularly to a depth of $0.3-0.5 \mathrm{~cm}$.

Patients in $\mathrm{C}$ group received 40 sessions of sham TAC conducted by the same acupuncturist. The acupuncture needle was pressed on but not inserted into non-acupoints of the tongue only. After a wash-out period of 2 weeks, the C group was crossed over to receive a course of TAC on the tongue acupoints.

\section{Outcome assessment}

The primary outcome measure was to assess for any change in gross motor function using a standardised scale (Gross Motor Functional Measure or GMFM). ${ }^{12}$ The secondary outcome measure assessed for any change in self-care, or social and cognitive function ${ }^{13}$ using the Pediatric Evaluation of Disability Index (PEDI). Subsequently, those in the C group were crossed over to receive TAC and were then assessed. Parents were asked to report any adverse effects. Thus, assessment was performed for both the $\mathrm{T}$ and $\mathrm{C}$ groups in weeks 0 and 9, and for the $C$ group another assessment was performed after completing a course of real acupuncture (that is, week 20).

\section{GMFM}

To examine whether GMFM scores had a normal distribution, the mean GMFM score were converted from an ordinal scale of $0-3$ to an interval scale by computing the percentage scores. In our study, we have compared the mean difference score between the $\mathrm{T}$ and $\mathrm{C}$ groups using independent sample $t$ tests. We also assessed the assumption of normal distribution by the normal plot method and the Q-Q plot method (fig 1); the difference in mean GMFM score was found to be normally distributed by both methods. The detrended normal Q-Q plot also showed a scattered distribution of the data, signifying that it was not skewed. The mean difference of GMFM conforms to normal distribution; GMFM is a well documented measure of therapeutic effect on gross motor function in CP children.

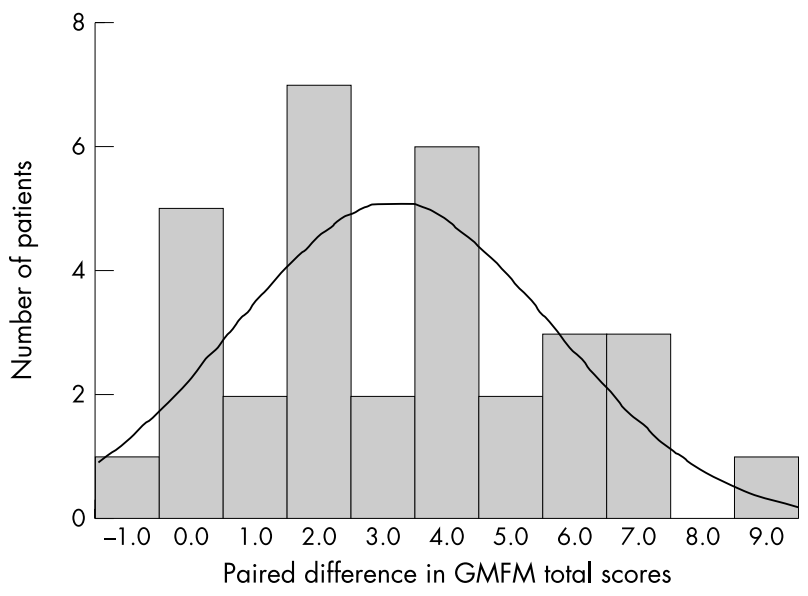

Figure 1 Normal plot of paired difference in GMFM total scores of treatment and control groups $(n=32)$.

\section{ROC curve analysis}

ROC (receiver operating characteristic) curve analysis demonstrated that the mean difference in GMFM score was dichotomised between the $\mathrm{T}$ and $\mathrm{C}$ groups (area under the curve (AUC) 0.727, $\mathrm{p}=0.037$ ) with the best cut-point at a mean difference of over 1.3 points (sensitivity 90.5\%, specificity $45.5 \%$ ). Analysis showed that 19/21 subjects in the $\mathrm{T}$ group improved by more than 1.3 points, compared to $6 / 11$ in the $C$ group. Five subjects in the $\mathrm{T}$ group improved from 5.9 to 9.0 points as compared to none in the control group.

\section{Statistical analysis}

SPSS version 9.0 for Windows was used for statistical analysis. Categorical data were analysed by the $\chi^{2}$ and Fisher exact tests. Inter- and intra-group differences were analysed with independent and paired samples $t$ tests. Significance was defined as being below a two-tailed probability of 0.05 .

\section{RESULTS}

\section{Treatment group: comparison of GMFM and PEDI} scores pre and post TAC

The mean GMFM score increased from 78.82 to 82.99 $(\mathrm{p}<0.001$, paired samples $t$ test $)$. For PEDI, only three scores achieved statistical significance: the mean PEDI self care subscore increased from 56.24 to 60.71 ( $p=0.001)$, the mean PEDI mobility subscore increased from 41.50 to 45.05 $(\mathrm{p}=0.027)$, and the mean PEDI self care assistance subscore increased from 26.48 to 31.33 ( $\mathrm{p}<0.001$ ).

\section{Control group: comparison of GMFM and PEDI scores} pre and post sham acupuncture

The mean GMFM score increased from 65.35 to 67.29 after a course of sham TAC $(p=0.022$, paired samples $t$ test $)$. No significant change was demonstrated in PEDI score.

\section{Comparison of paired differences of mean GMFM and PEDI scores in both the $\mathrm{T}$ and $\mathrm{C}$ groups (table 1 )}

The increase in mean GMFM score was significantly higher for the $\mathrm{T}$ than the $\mathrm{C}$ group $(\mathrm{p}=0.042$, independent samples $t$ test). The mean differences in GMFM scores were also statistically significant when analysed with non-parametric tests $(p=0.039$, Mann-Whitney U test). For PEDI, only a marginal significantly increase in the PEDI self care subscore for the $T$ group was seen $(p=0.054)$.

In the $\mathrm{C}$ group after crossing over to TAC treatment, the paired differences (mean (SE)) in GMFM scores (compared with baseline scores) after sham or TAC were $1.94(0.72)$ and 4.29 (1.02), respectively $(\mathrm{p}=0.008$, paired samples $t$ test $)$. However, no significant difference was demonstrated in the paired differences of the PEDI subscores.

All but one child completed the study. A child in the control group withdrew from the trial after crossing over to receive genuine TAC, dropped out after the 25th session of TAC, and did not return for re-evaluation of GMFM scores. However, his parents returned the PEDI for analysis.

\section{Side effects}

The TAC procedure was tolerated by most children. Initial crying with fear and possible minor pain occurred in the first few sessions. Subsequently, most patients became adapted and more compliant.

\section{DISCUSSION}

This is the first randomised control trial of the acupuncture in $\mathrm{CP}$. We have demonstrated the efficacy of an innovative and relatively non-invasive method in short-term improvement in gross motor function in CP. 
Table 1 Comparison of paired differences (post-acupuncture scores minus baseline scores) in GMFM subscores between treatment and control groups

\begin{tabular}{llllll}
\hline & $\begin{array}{l}\text { Treatment group, } \\
\text { paired difference } \\
\text { (SE) }\end{array}$ & $\begin{array}{l}\text { Control group, } \\
\text { paired difference } \\
\text { (SE) }\end{array}$ & $\begin{array}{l}\text { Mean } \\
\text { difference } \\
\text { (SE) }\end{array}$ & 95\% Cl & p \\
\hline Lying and rolling & $1.51(0.82)$ & $0.89(1.03)$ & $0.62(1.36)$ & -2.15 to 3.39 & 0.649 \\
Sitting & $2.11(0.58)$ & $2.26(0.72)$ & $-0.15(0.96)$ & -2.11 to 1.81 & 0.877 \\
Crawling and kneeling & $4.49(1.85)$ & $2.82(1.15)$ & $1.67(2.65)$ & -3.74 to 7.08 & 0.534 \\
Standing & $3.47(0.94)$ & $1.87(1.39)$ & $1.59(1.63)$ & -1.74 to 4.92 & 0.336 \\
Walking, running, and & $6.00(1.55)$ & $1.77(1.51)$ & $4.23(2.37)$ & -0.62 to 9.09 & 0.085 \\
jumping & & $1.94(0.72)$ & $1.86(0.88)$ & 0.07 to 3.66 & 0.042 \\
GMFM total score & $3.80(0.51)$ & & & & \\
\hline
\end{tabular}

In our study, the treatment group showed a significant increase in GMFM, PEDI self care, PEDI mobility, and PEDI self care assistance subscores after 40 sessions of TAC. However, a modest but statistically significant increase in GMFM scores was also found in the control group, which may be related to increased ambulation during the treatment phase. This possible effect of ambulation was eliminated by the relatively greater increase in GMFM scores in the treatment group. Furthermore, children in the control group who subsequently crossed over to receive genuine TAC also demonstrated a significant increase in GMFM scores. Our results thus reflect the beneficial effect of short-term TAC on the gross motor function and self care ability of CP children.

Significant improvement in gross motor function was demonstrated by the GMFM but not the PEDI scale. However, the GMFM and PEDI scales measured different aspects of motor performance. The GMFM is designed to measure the best-ever performance in a particular test situation upon instruction, and is not a measure of the quality of performance the child may manifest in daily life. ${ }^{12}$ In contrast, the PEDI measures the essential functional skills and the capacity to perform complex activities in daily life in the child's environment. ${ }^{13}$ Thus, this could also explain the lack of significant difference in improvement in PEDI scores in both groups.

For the GMFM, none of the subscores' paired differences were significantly different between the two groups, but that of the total score was significantly different $(p=0.042)$. Nonetheless, we observed a trend of decreasing $\mathrm{p}$ values as we moved up from the GMFM (lying) to GMFM (walking) subscores, while the $95 \%$ CI shifted gradually to a positive range (though still including zero) (table 1). This trend might reflect the fact that those $\mathrm{CP}$ subjects who could stand or walk at baseline might gain most from TAC, though our small sample size did not have adequate power to demonstrate a statistical difference.

The heterogeneous mix of CP children with different abilities might further "dilute" the true difference. Nevertheless, this pilot study served to guide us in selecting patient to receive acupuncture therapy. Further study may focus on a more homogeneous group of ambulatory CP children whose functional improvement from conventional treatment has already plateaued off.

Acupuncture increased activity in the thalamic nuclei, neocortex, and limbic lobe of the cerebral cortex of rat ${ }^{14}$ and also stimulated the hypothalamus and brainstem, thus influencing the sympathetic nervous system. ${ }^{15}$ The clinical efficacy of acupuncture had been postulated to be based on improvement in cerebral blood flow, improvement in brainstem function, or stimulation of the adrenocortical system. ${ }^{5}$

Our pilot randomised control trial of CP children demonstrated short-term functional gain in children with chronic motor dysfunction. Acupuncture has a potential role in improving the functional status of the patient within a short period. This functional gain may then be consolidated by conventional physiotherapy. TAC may thus be used as an adjunctive therapy to anti-spasticity procedures.

More research into the mechanism of neuroplasticity following this "old traditional and alternative" treatment regime is definitely needed. Further studies on the physiological effects of acupuncture in the brain are required. The optimal time, frequency, and dosage of treatment need to be determined, as well as the sustainability of any therapeutic effect after each course of treatment. In the future, randomised control trials of acupuncture courses with larger sample sizes and longer follow-up (if repeated) are required to examine the role of acupuncture as an adjunctive therapy in the integrated management of $\mathrm{CP}$ with conventional Western modes of therapy.

\section{ACKNOWLEDGEMENTS}

We would like to thank SM Yuen (nurse coordinator), A Ng (physiotherapist), and W Wong (statistical advice) for assistance in this project.

\section{Authors' affiliations}

J G Sun, The Jockey Club MRI Engineering Center, The University of Hong Kong, Hong Kong, China

C H Ko, Department of Pediatrics, Caritas Medical Center, Hong Kong, China

V Wong, Department of Pediatrics and Adolescent Medicine, The University of Hong Kong, Hong Kong, China

X R Sun, Liaoning Tongue Acupuncture Research Institute, Liaoning, China

Competing interests: none declared

Correspondence to: Professor V Wong, Division of Neurodevelopmental Paediatrics, Department of Pediatrics and Adolescent Medicine, The University of Hong Kong, Hong Kong, China; vcnwong@hkucc.hku.hk

Received 24 July 2003

In revised form 25 November 2003

Accepted 8 December 2003

\section{REFERENCES}

1 Graham HK, Aoki KR, Autti-Rämö I, et al. Recommendations for the use of botulinum toxin $A$ in the management of cerebral palsy. Gait Posture 2000;11:67-79.

2 McLaughlin J, Bjornson K, Temkin N, et al. Selective dorsal rhizotomy: metaanalysis of three randomized controlled trials. Dev Med Child Neurol 2002;44(1):17-25.

3 Coffey RJ, Cahill D, Steers W, et al. Intrathecal baclofen for intractable spasticity of spinal origin: results of a long-term multicenter study. J Neurosurg 1993;78:226-32.

4 Hsu DT. Acupuncture. Reg Anesth 1996;21:361-70.

5 Filipowicz WA. The application of modern acupuncture techniques and methods on children with cerebral palsy. Am J Acupunct 1991;1:5-9.

6 Shi BP, Bu HD, Lin LY. A clinical study on acupuncture treatment of pediatric cerebral palsy. J Trad Chin Med 1992;12:45-51. 
7 Lu WQ. Prompt pressure applied to peculiar points in the treatment of spasmodic infantile cerebral palsy-a report of 318 cases. J Trad Chin Med 1994; 14:180-4

8 Zhou XJ, Chen T, Chan JT. 75 infantile palsy children treated with acupuncture, acupressure and functional training. Chung-Kuo Chung His I Chieh Ho Tsa Chih 1993;13:220-2.

9 Sanner G, Sundequist U. Acupuncture for the relief of painful muscle spasms in dystonic cerebral palsy. Dev Med Child Neurol 1981;23:544-5.

10 Svedberg LE, Nordahl UEG, Lundeberg TCM. Effects of acupuncture on skin temperature in children with neurological disorders and cold feet: an exploratory study. Complement Ther Med 2001 ;9:89-97.
11 Wong V, Sun JG, Wong W. Traditional Chinese medicine (tongue acupuncture) in children with drooling problems. Pediatr Neurol 2001;25:47-54 12 Russell D, Rosenbaum P, Gowland C, et al. The Gross Motor Function Measure Manual, 2nd ed. Hamilton, ON: McMaster University, 1993.

13 Haley SM, Coster WJ, Ludlow LH, et al. Pediatric Evaluation of Disability Inventory (PEDI). Version 1. Development, standardization, and administration manual. Boston, MA: New England Center Hospital, 1992

14 Futaesaku Y, Zhai N, Ono M, et al. Brain activity of a rat apparently reflects the stimulation of acupuncture. Cell Mol Biol 1995;41:161-70.

15 Guillemin R, Vargo T, Rosier J, et al. Beta-endorphin and adrenocorticotropin are secreted concomitantly by the pituitary gland. Science 1977;197:1367-9

\section{NEURONLINE}

\section{Brainlnfo: http://braininfo.rprc.washington.edu}

Q uoting from the website, "BrainInfo is a website that helps one identify structures in the brain and provides many different kinds of information about each structure. It consists of three basic knowledge bases: Neuro Names, which provides the index to brain structures and narrative information about them; the Template Atlas, which shows the structures that are found in the primate brain; and NeuroMaps, a set of several hundred overlays that will show the location of different kinds of information that have been mapped to the standard background maps (templates) of the Atlas. Information about brain structures in other species, particularly the human, is provided by links to other websites."

This website has been set up to help negotiate the user through the adult mammalian brain. This is achieved using a series of different selection criteria, such that on entering the area of brain that you are interested in, you can then explore a whole range of different aspects of that structure. This includes its location using a battery of different pictures including radiological and anatomical figures, which are enormously helpful for clinicians and neuroscientists alike given the links to human brain images. Indeed ones neuroanatomical quest can be further expanded to explore the cell types within the area of interest and even the receptors that are expressed on these cells. In addition the site also provides all the different nomenclatures that may be used to describe that structure or parts of it and, finally, you can then link across to PubMed for references related to that structure.

Thus, this website is an extremely powerful resource, but as with many such sites the problem can be in trying to extract the major, significant "bytes" of information. Therefore, most of us would know the major afferent and efferent connections of structures within the CNS, which are useful for our clinical and scientific work, without

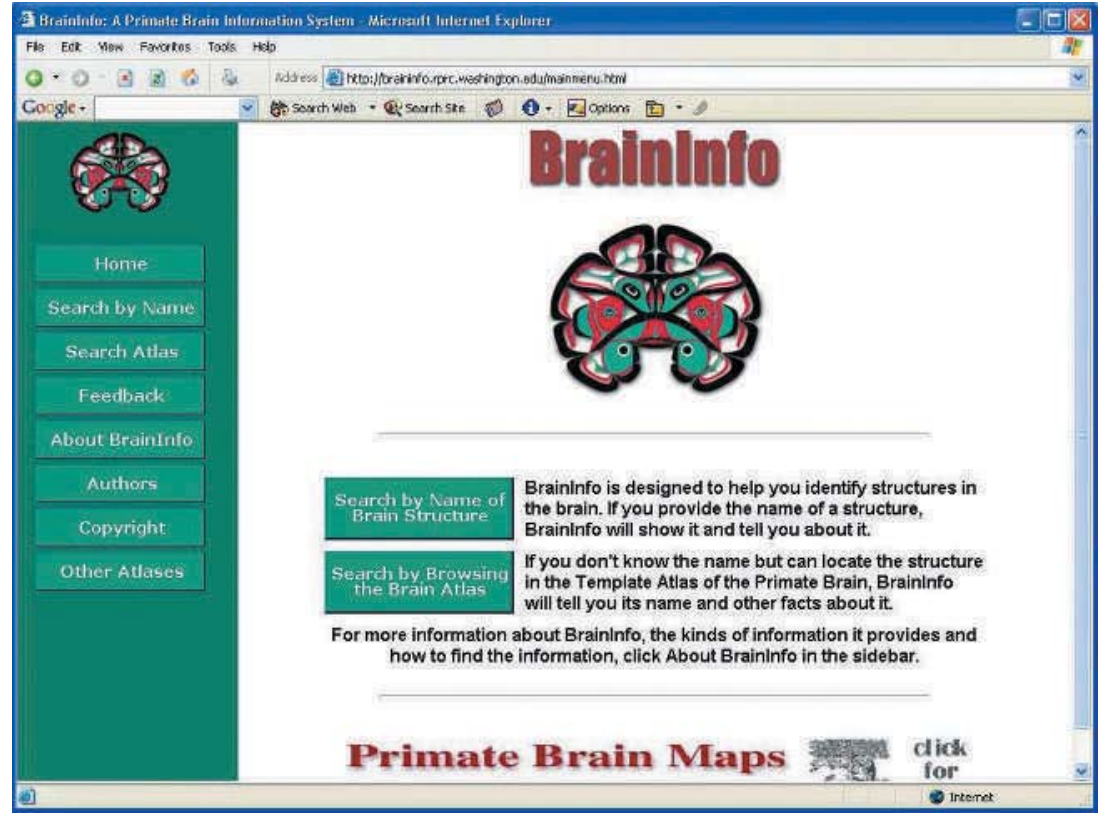

needing to know the finer details. So, for example, the substantia nigra has a series of well known connections with the striatum, pallidum, superior colliculus, and the thalamus, which allows us to understand crudely its role in basal ganglia function. However, the comprehensive nature of the data presented on this website lists 16 confirmed afferent and 21 efferent projections of the substantia nigra along with a range of further inferred connections! To the novice this is rather overwhelming and baffling, and so to make sense of this one often needs to be educated by less rigorous neuroanatomical accounts that are found in standard neuroscience textbooks.

Nevertheless, this site is very helpful in locating those areas that passed by you during neuroanatomy lectures and practicals, while allowing the specialist to investigate in great detail the brain region and even the cell of interest. All this information is presented in a user friendly fashion with a mass of references for those wanting confirmation of the origin of the data as well as further reading. Indeed, you even have the capacity to add your own comments and with that you can add even more relevant references! Overall this is a very useful website to know about and I have certainly added it to my bookmark list.

R T A Barker Cambridge Centre for Brain Repair and Department of Neurology, Addenbrooke's Hospital, Hills Road, Cambridge CB2 2PY, UK; rab46@hermes.cam.ac.uk 